\title{
1 Predicting Non-Residential Building Fire Risk Using Geospatial 2 Information and Convolutional Neural Networks
}

3 Jake Anderson-Bell ${ }^{\text {a }}$, Calogero Schillaci ${ }^{\mathrm{b} *}$, Aldo Lipani ${ }^{\mathrm{a}}$

$4{ }^{a}$ Department of Civil, Environmental and Geomatic Engineering, Gower Street,

5 London, University College London

$6{ }^{b}$ Department of Agricultural and Environmental Sciences, Milan Via Celoria 2,

7 University of Milan

8 *calogero.schillaci@unimi.it

9 Abstract

10 Building fire risk prediction is crucial for allocation of building inspection 11 resources and prevention of fire incidents. Existing research of building fire 12 prediction makes use of data relating to local demography, crime, building use and 13 physical building characteristics, yet few studies have analysed the relative 14 importance of predictive features. Furthermore, image features relating to 15 buildings, such as aerial imagery and digital surface models (DSM), have not been 16 explored. This research presents a multi-modal hybrid neural network for the 17 prediction of fire risk at the building level using the London Fire Brigade dataset. 18 The inclusion of traditional and novel image features is assessed using Shapley 19 values and an ablation study. The ablation study found that while building use is 20 the most effective contributor of classification performance, demographic features, 21 apart from social class, are detrimental. Moreover, while the DSM did not lead to 22 any significant improvement in classification performance, the inclusion of the 23 aerial imagery feature lead to a $4 \%$ increase in median validation ROC AUC. The 24 final model presented achieved an ROC AUC of 0.8195 on the test set.

\section{Introduction}

Fire related incidents impact human communities across the globe, posing a threat to

27 human life, damaging property, and hindering productivity in their wake. Recent advances in fire safety have aided a decrease in the total number of fires in the United 
29 Kingdom from 473,000 in 2003/04 to 162,000 in 2016/17 (Bryant and Preston, 2017).

30 While more effective fire safety and incident response systems have brought total 31 casualties down, the costs associated with property loss due to fire have remained high since 1990 in the United States, residing at a value of \$13.2 billion as of 2014 (Zhuang et al., 2017). Due to such cost associated with fire, municipal fire brigades, such as The City of Pittburgh's Bureau of Fire (Madaio et al., 2018) conduct inspections on properties to assess properties deemed to have a high risk of fire. Owing to the high number of potential properties it is not possible to carry out inspections on all buildings on a regular basis (Pringle and Welsh, 2015). Approaches to inspection allocation, employed throughout the world, rely on the analysis of the relationship between building fire incidents and potential determinant variables, ranging from physical building characteristics to 40 sociodemographic factors, to focus efforts where they are needed. For this reason, numerous studies have focussed on finding effective means by which properties at high risk of fire can be more readily identified (Dang et al., 2019; Walia et al., 2018). By adopting such methods, fire brigade services can reduce their operational costs and more

44 fires may be prevented. Although several studies have employed data regarding fire inspection, demography, and commercial information to classify non-residential building fire risk, the use of aerial imagery and digital surface models (DSMs) was rarely explored.

47 Furthermore, while the datasets used are effective in their task, little research has investigated these variables as individual contributors to classification performance.

49 Risk prediction models have been implemented extensively in a range of subject areas, 50 such as medicine (Preuschoff, Quartz and Bossaerts, 2008) and economics (Kuester et al., 2006). Although academic work has been dedicated to the task of predicting nonresidential building fire risk, there has been a relative lack of studies that explore feature impact on classification performance and none have explored the use of aerial imagery 
and DSMs as features. In the context of machine learning ML, the term feature denotes a variable that is used to predict an output.

To focus fire risk inspection efforts to areas within a city where risk of fire a greater, some studies have grouped areas of higher building fire incidence together and treated them as a whole. In a randomized control trial with Surrey Fire Services, British Columbia, Claire et al. (2012) indicated zones of high residential building fire risk within the study area for a smoke alarm installation initiative. They then mapped residential structure fires as point features before a series of ellipses were drawn to capture points within zones containing high concentrations. A drawback of such an approach is the subjective nature of the methods. In contrast, DaCosta et al., (2015) joined American Housing Survey and American Community Survey datasets then used a Random Forest to model residential fire risk at census block level. Such a method, whereby regional open data is aggregated will also be adopted in this study. Increasingly, studies concerned with prediction of fire risk are looking to a more granular approach where individual building fire risk is considered. Garis and Clare (2014) developed a commercial building fire risk framework based on physical building characteristics in conjunction with history of fire regulation compliance. Although this system is heavily based on specifics of local fire safety regulation, their method builds a systematic approach to fire safety inspection resource allocation. To prioritise fire inspection to higher risk commercial properties, Madaio et al., (2015) joined historical fire incident and inspection records at the building-level with U.S. census data, such as age, ethnic population, and income, at a more regional level. They also used crime incident records and Google Place API data to get up to date business information. The area under curve (AUC) for true positive rate against false

77 positive rate obtained from random forest and SVM models was 0.813 and 0.805 , 
classes. Although this research refers to data sources used, the features themselves are

80 not described in detail. The current study draws some parallels from Madaio et al., (2015)

81 in that Google Places API and demographic data will be used to gather information to aid

82 fire risk predictions at the building scale.

83 Madaio et al., (2015) briefly touch on feature importance in their modelling. They find

84 that for the random forest model, features relating to building size and its physical

85 characteristics have the greatest impact on their output. In contrast, they then construct a logistic regression model and analyse feature coefficients which suggest that the Google Places feature has the greatest importance. Dang et al. (2019) used data provided by Humberside Fire and Rescue Service regarding property inspections and fire severity in conjunction with publicly available open data on food hygiene and Google Places ratings,

90 among others, to build a commercial fire risk model. They experiment with several 91 different learning algorithms.

92 While the multi-layer perceptron model they implemented attained an area under curve 93 (AUC) score of 0.78 , an AUC score of 0.89 for an XGBoost model was also achieved.

94 Although this study represents the highest performing classification of commercial 95 building fires in the literature, it does not give much consideration to analysis of features as individual contributors to classification performance.

97 Whilst there is some crossover in mutually used features in Madaio et al., (2015) and

98 Dang et al., (2019), there is little rationale given for features used and attempts made to 99 analyse the classification performance contributions of individual features are 100 inconsistent within studies. In contrast to using a feature set that includes demographic 101 variables, Hong and Jeong (2018) conducted a study whereby 16 features relating to 102 physical building characteristics and fire history were used to make fire predictions of 103 fire risk. Data was used to train support vector machine SVM, Nave Bayes, Decision tree 
104 and artificial neutral networks ANN models. The SVM had highest test set accuracy

105 overall at $63.54 \%$. While many features used by Hong and Jeong (2018) relating to

106 physical building characteristics were not available for this study, it is hypothesised that

107 the use of aerial imagery and DSMs may supplement physical features that they used.

108 Although this study is only concerned with predicting fire risk for buildings, by using

109 features based on aerial imagery, similarities are shared with studies in wildfire prediction

110 and land use classification (Collins et al., 2020; Oliveira and Zêzere 2020), building

111 assessment (Monfort et al., 2019), potential of rooftop solar energy (Schunder et al.,

112 2020). Mitri et al., (2015) used pixel segmentation and subsequent object classification

113 to determine presence of different wildfire fuels (e. g. grass, shrub, etc.) to discover areas

114 susceptible to combustion.

115 In ML a hybrid model is a combination of two or more existing algorithms to produce a

116 single output that can make use of different forms of data related to the same task. Such

117 architectures have been implemented using CNN models for multiple image features

118 (Wang et al., 2018) and ANN models for features of varying structure (Audebert et al.,

119 2019). This method has advantages over running models separately as the error can be

120 calculated over the entire network. Audebert et al., (2019) trained a hybrid model for

121 classification of documents from image and textural input which attained a higher

122 performance than the two separate models. A hybrid model will be implemented in this

123 study to make use of multiple features of varying formats for each training example.

124 Convolutional neural networks (CNN) for risk-based classification of image data have

125 been used more widely in the field of medical research than any other subject. Wang et

126 al., (2018) uses three image features of lung scans to predict the malignancy risk of

127 pulmonary nodules through use of a CNN. In their methodology they compare a hybrid

128 CNN (whereby three image inputs per training example are inputted to a multi-branch 
$129 \mathrm{CNN}$ ) with a multi-channel fusion $\mathrm{CNN}$ (where three image inputs are layered on of one

130 another to produce a single tensor then propagated through a single-branch $\mathrm{CNN}$ ). They

131 achieve multi-channel and fusion channel AUC or 0.93 and 0.97 , respectively. Although

132 Wang et al., (2018) achieve a greater AUC on the fusion-channel model, the multi-branch

133 CNN approach makes more sense for this study as the inputs require merging with

134 additional dimensionally dissimilar tabular data before a final classification.

135 Urban street view imagery has been successfully implemented in several studies to extract 136 useful information about the built environment. Liu et al. (2017) used street view imagery

137 to classify construction and maintenance quality of buildings in Beijing. The model they

138 produced achieved an F1 score of $61.8 \%$, suggesting that computer vision can be

139 implemented to classify quality of buildings to some extent. Similarly, Law et al., (2018)

140 produced a CNN model that classified street view imagery by aesthetic street frontage

141 quality. Furthermore, Law et al., (2018) used aerial imagery in conjunction with street

142 view imagery to estimate house prices. This research relates to these studies in that an

143 attempt will be made to gain information regarding the built environment through

144 computer vision.

145 Numerous studies have investigated classifying roof types of buildings. In a study

146 concerned with building detection and roof type classification from aerial imagery

147 Alidoost and Arefi (2018) used labelled instances of roof types to achieve a classification

148 accuracy of $92 \%$ with a CNN model. Such research suggests that ML algorithms have the

149 potential to classify areal characteristics of buildings.

150 Our study will address this gap in the literature by exploring the use of aerial imagery and

151 DSMs, and assess the relative importance of these in addition to traditional variables for 152 non-residential building fire risk classification using CNN. Moreover, we assess the 153 ability of traditionally used features in building fire risk prediction. We do this using a 
154 feature attribution method, named Shapley values, to understand the importance of

155 feature values towards the prediction; and conduct a feature ablation study to assess the 156 impact of each individual feature.

\section{Material and methods}

\subsection{Data}

159 The following section details the procedure taken and rationale behind the

160 implementation in the data pre-processing and methodological stages of the study. Scripts

161 used in this research can be found in a Github repository and accessed via the URL in supplemental materials. The tabular data included in the predictive modelling of nonresidential building fire risk was chosen due to them being included in previous studies.

164 This allows better comparison of the results of this work with other models constructed

165 in the literature. While some data sets like those used previously have been implemented

166 here, data availability has been a constraining factor. Many variables used in this study

167 have been attributed to buildings via spatial joins, summarised in Figure 1. An assumption

168 of this method is that data collected for a building is representative of that building, the

169 people who use it and the processes that occur within or around it. Furthermore, it is

170 assumed that data of a more regional resolution that is attributed to individual buildings

171 is also representative of the social characteristics at a local level.

172 Due to the investigation of the inclusion of imagery and surface models in prediction

173 being a central aim of this study, the approach chosen has been influenced by methods

174 where image data may be an input to the model. An ANN was chosen as the algorithm to

175 be used as they have been successfully implemented in recent years in the field of image

176 classification and can accept multiple data types as inputs to the model (e.g. tabular data

177 and imagery) (Geiß et al., 2020; Kim et al., 2020a, 2020b). 


\section{Output Area - ONS Data}

Building Shape

- Aerial imagery

- DSM

- Google Places

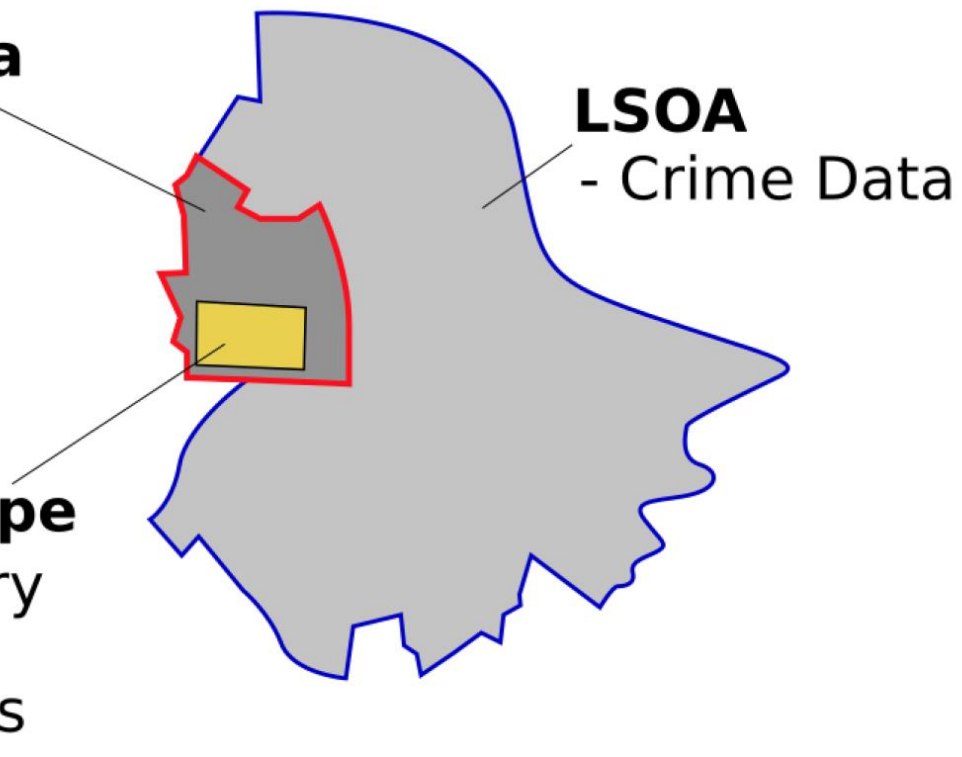

179 Figure 1: Diagram illustrating how data from varying levels of spatial attribution have

180 been merged to represent individual buildings, LSOA = Lower super output area

\subsubsection{London Fire Brigade Incident Data}

The London Fire Brigade (LFB) provide information regarding all fire incidents reported from 2009 to present (London Fire Brigade, 2011). This was used in order to find locations of fire incidents. The accuracy to which the incident location is recorded varies within the dataset. This is recorded with an address qualifier variable that states, for example, whether the incident location is correct or accurate to the street on which it occurred. When the proportion of building category fires in the dataset is compared to the proportion of building category fires recorded at the correct incident location (figure 1) it can be seen that there is a bias towards collecting the correct location information for nonresidential fires. Although only non-residential building fires are the subject of this study, this bias suggests that there are some inconsistencies within the ways that the data is collected. While this is only apparent in a contrast between how residential and nonresidential buildings are handled, it is not clear whether there exists a bias within the way that non-residential building fire locations are recorded. 
Property type as proportion of all fire incidents

195

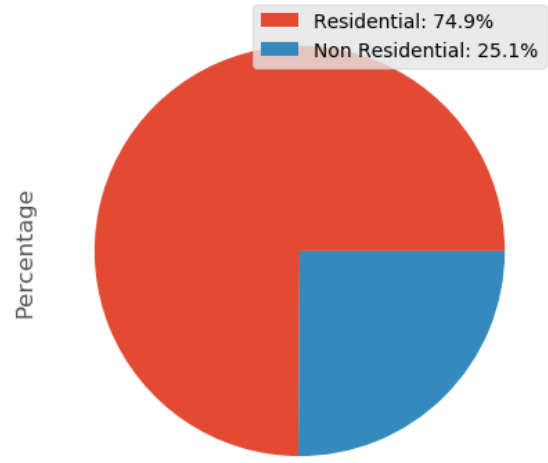

Property type as proportion of correct incident location incidents

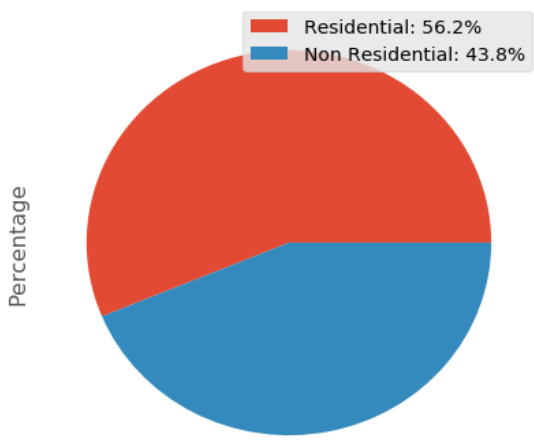

196 Figure 2: Proportions of property types for (a) all fire incidents in dataset and (b) fire incidents

197 with correct location

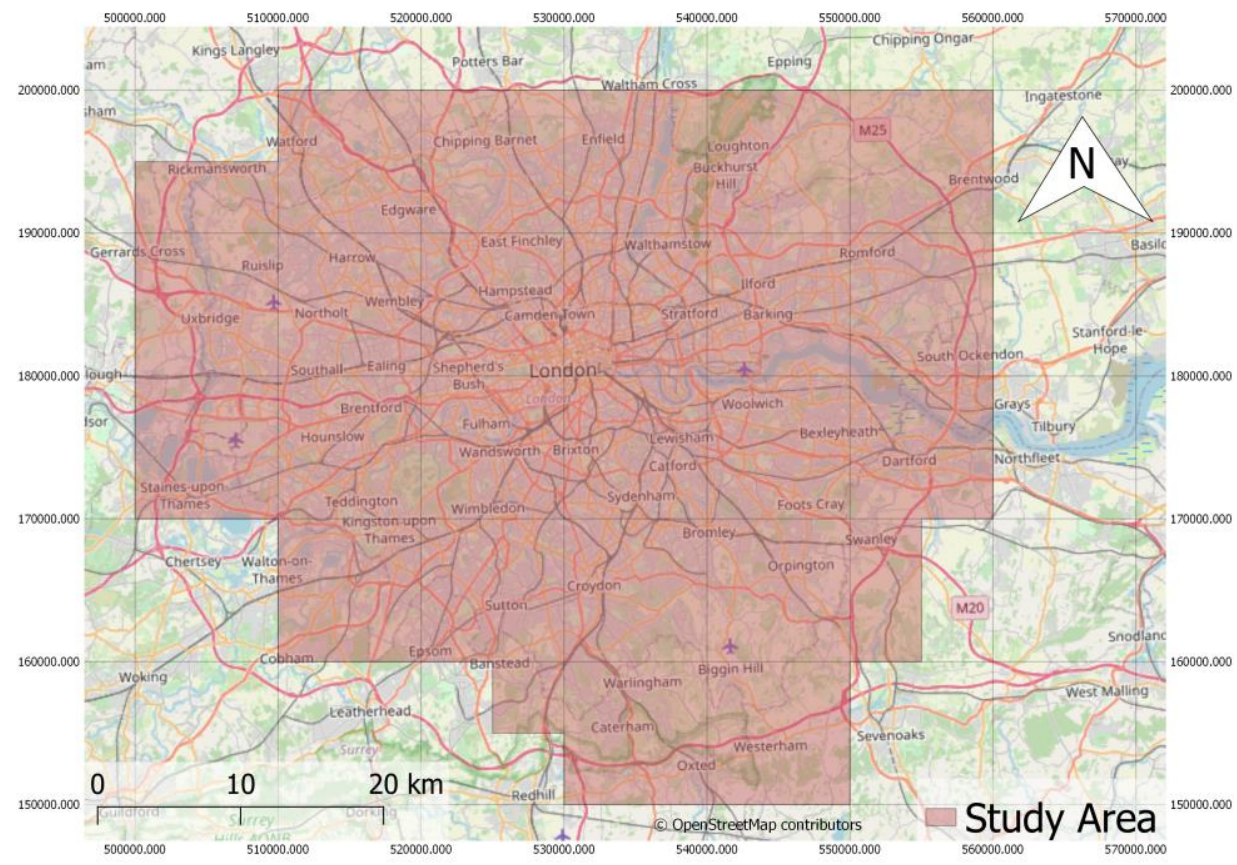

199 Figure 3: Study area used in this investigation, Greater London

200 The geographic study area is based on the spatial extent of the LFB dataset. This covers

201 the area of Greater London and is presented in figure 3.

203 This study is concerned with fires whose severity may have been impacted by building

204 design. For this reason, all fires in the dataset were filtered for those that were primary 205 fires. Primary fires are generally more serious and caused more damage than other 206 categories of fire (Home Office, 2020). The address qualifier variable, stating to what 
207 degree of accuracy the incident location is recorded was used to filter incidents for those

208 that are recorded at an accuracy of being in the correct building or greater. The location

209 of the incidents were recorded in British National Grid, which was used to convert the

210 tabular data into point features.

\section{2.1.2 Ordnance Survey MasterMap}

212 Ordnance Survey MasterMap (MM) is a database of shapefiles recording every fixed

213 feature larger than a few metres in Great Britain (Ordnance Survey, 2020). This data was

214 used to obtain the shapes of buildings used for clipping aerial imagery and DSMs. This

215 data was accessed and downloaded from Digimap (Edina, 2019). Multiple versions of

216 MM were been downloaded to match the annual extents of the aerial imagery.

217 MasterMap shapefiles covering the area of Greater London were collected for all years

218 available within the timespan of the London Fire Brigade (LFB) dataset. After being

219 filtered for buildings, LFB incident points were grouped annually, and a spatial join was

220 performed between the LFB points and the version of MM building polygons closest to

221 the time of the LFB incidents. All points that did not fall within a building shapefile were

222 eliminated from the data set.

\section{$223 \quad 2.1 .3$ Aerial Imagery and Digital Surface Model}

224 The vertical aerial imagery used in this study was obtained using EDINA Aerial Digimap 225 Service (Edina, 2019) and collected by Getmapping (Getmapping, 2019). Although 226 satellite imagery may have been available for use in this study, the flight captured imagery

227 had a resolution of $25 \mathrm{~cm}$ ground sample distance (GSD), greater than any other open 228 aerial imagery data available at the time this research was conducted. 


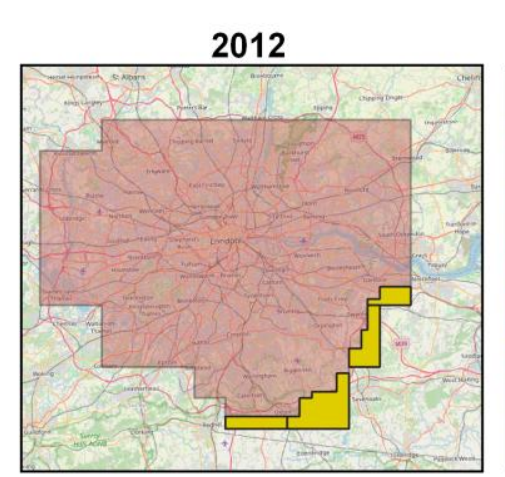

2015

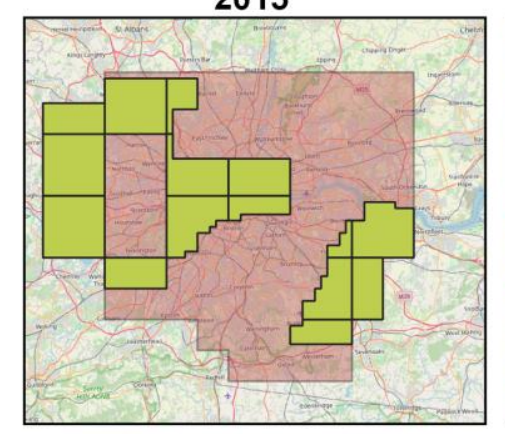

2018

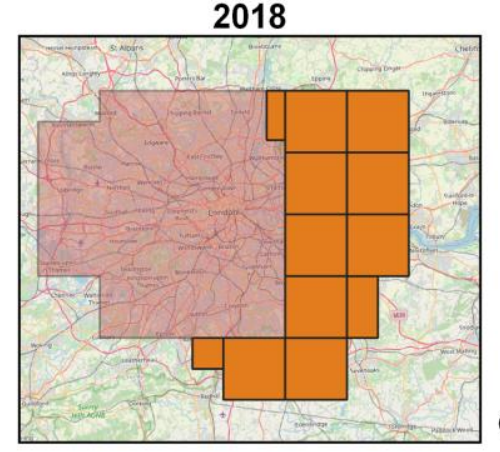

2013

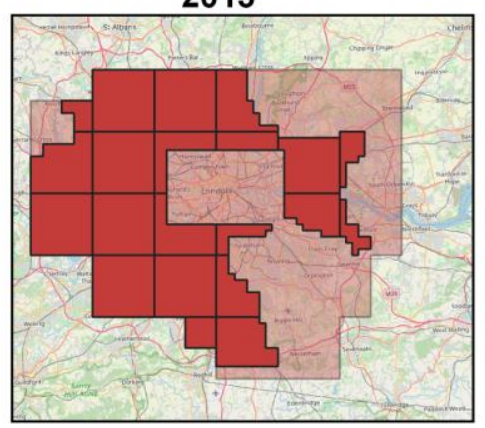

2016

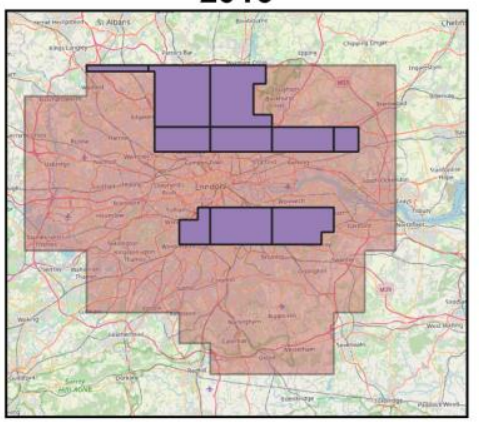

2014

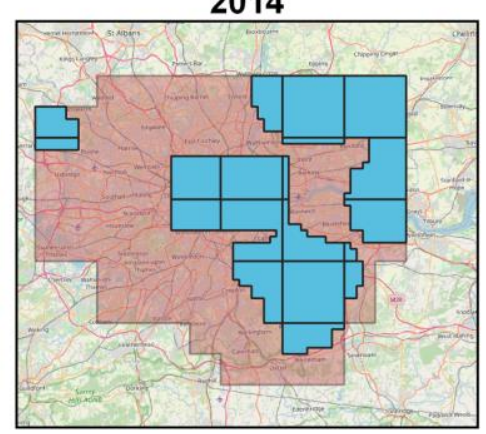

2017

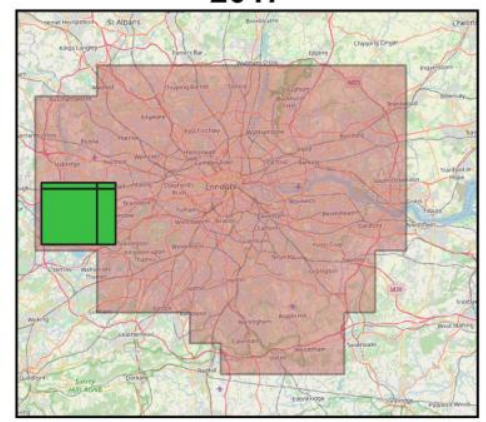

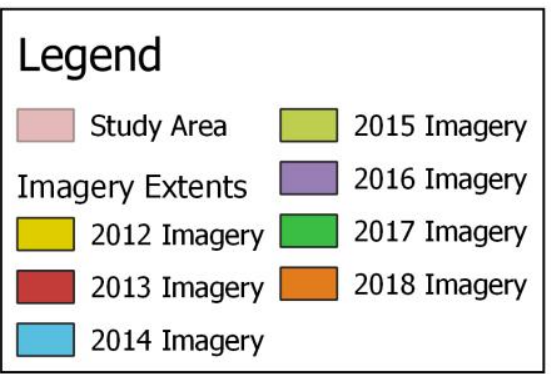

(c) OpenStreetMap contributors

230 Figure 4: Annual spatial coverage of aerial imagery used in the study (10km tile grid)

232 Aerial imagery used was collected between 2012-2018, however different extents of the

233 study area were collected each year (figure 4), with each point on the ground being

234 revisited every 3-4 years. Georeferencing had already been performed on the imagery and

235 this was provided in the form of JGW files. The imagery was not orthorectified so some

236 building lean exists in the data. The digital surface models were accessed from EDINA

237 DigiMap (Edina, 2019) and consist of a LiDAR composite digital surface model. The

238 data was collected by the Environment Agency from 2006 onwards and is available under 
239 the Open Government Licence for public sector information (Environment Agency,

240 2020). The dataset has a vertical accuracy of $\pm 15 \mathrm{~cm}$ RMSE and a spatial accuracy of

$241 \pm 40 \mathrm{~cm}$ RMSE (Environment Agency, 2020). As the resolution of the dataset is $1 \mathrm{~m}$,

242 greater than the mean spatial error, the spatial error has negligible impact in this

243 application.

244 Imagery metadata was used to determine the date that each image was taken. Because the

245 appearance of, or even the buildings themselves, can change over time it was decided that

246 only imagery that was taken within a year prior to a fire incident would be used. The

247 recorded incident date was used to find relevant imagery. In contrast, the DSM was much

248 more mixed in terms of when it was collected and processed, for instance some individual

249 tiles have data collection spanning several years so it is difficult to determine the exact

250 time in which the DSM for a building was collected. While building structures do change

251 over time, they are not as variable as the appearance of buildings and so the time of DSM

252 was not considered in this study. 


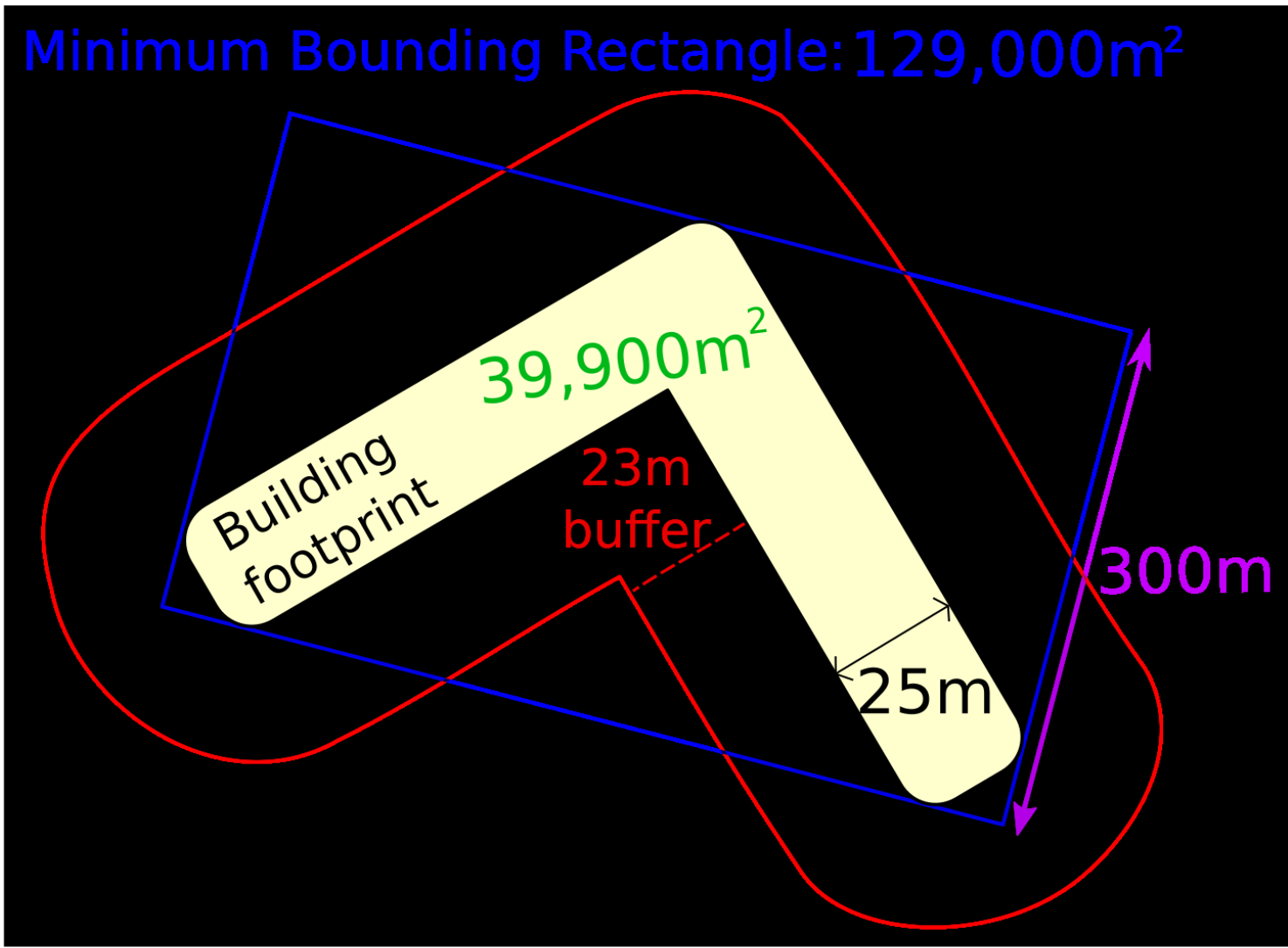

Figure 5: Diagram showing how data regarding building shape is used to calculate a scale and shape complexity variant buffer distance with equation (1), Pink=MRA shortest dimension, Green=Shape area, Blue=MRA, Red=Buffer distance .

Building footprint $* 0.25 * 300(39900 / 129900)=23$

261 The area surrounding a building may also have an impact on its fire risk, so a buffer was made around the building before clipping the imagery. A building size dependent buffer amount was chosen due to variation in building size within the dataset. When using a buffer size proportionate to the area of the building or the bounding box of the shape, complex or narrow and branching building shapes became dominated by the buffered

266 area (Figure 5). A method was required for the final images to be representative of the

267 building, whilst also considering the surrounding features.

268 The following rule was applied. 
270 Where BD is the buffer distance, $\mathrm{A}$ is the area of the shape, MRA is the area of the

271 minimum bounding rectangle of the shape, and $\mathrm{S}$ is the shortest dimension of the

272 minimum bounding rectangle. This method is summarised in figure 6 .

273 When the building is a rectangle this results in a buffer size of a quarter of its shortest

274 dimension, however, with more complex building shapes with more open space within

275 their bounding boxes, the size of the buffer becomes moderated by the ratio between the 276 area and the minimum bounding rectangle area.

277

278
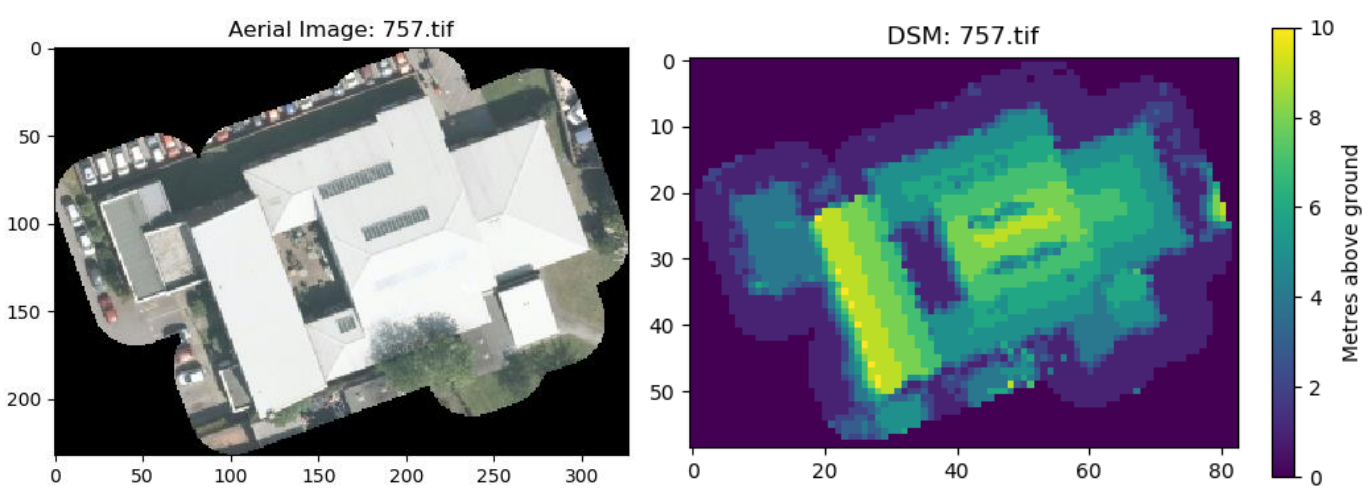

279 (a) Aerial imagery, left panel (b) Digital surface model, right panel

280 Figure 6: Example of aerial imagery and DSM data after clipping (Getmapping, 2019;

281 Environment Agency, 2020).

282

283 The building shapes were then buffered with a buffer size from the equation (4.1) and this

284 was used to clip the aerial imagery and DSM to image files (figure 6). The minimum

285 height of the DSM for each building was then subtracted from each file so that the DSM

286 was relative to the ground level in each instance. The aerial imagery was greyscale

287 sampled to produce a 2D feature. The regions of the aerial images and DSM that fell outside of the buffer were given a pixel value of 0 . 


\subsubsection{Google Places}

290 The Google Places API is a tool implemented in this study to obtain information regarding

291 businesses and services that occupy a building (Google, 2020). In addition to finding

292 information about the buildings in which incidents occurred, Google Places was also used

293 to find commercial data for spatially random buildings where fires did not occur. Using

294 the service involves sending a request with a query to the Places server before receiving

295 a response of 20 results per request. Requests can be made by place name, address, or by

296 location and can be filtered by type of place.

297 To get the business information about the buildings in the fire incidents dataset, a request

298 was made for each MM building centroid linked to a fire incident. A radius option,

299 whereby places returned whose location falls within were prioritised in the request, was

300 set at $100 \mathrm{~m}$. Returned places results were searched for businesses whose location was

301 within the building shapes. The building, incident and place data were then joined. The

302 Places API was also used to find commercial buildings to be used for the

303 negative fire incident dataset (i.e. those where a fire did not occur). To attempt to have

304 the negative classes to have a similar spatial distribution to the positive class, the

305 frequency of fire incidents in each $10 \mathrm{~km}$ tile was calculated. These were then used as the

306 basis for the quantity of negative classes to find in each $10 \mathrm{~km}$ tile. For each tile, a series

307 of random points was generated and used to make Places API requests. The results were

308 then searched for places whose location fell within buildings where no fire incident was

309 recorded. This served as the foundation for the negative fire examples for which aerial

310 imagery and DSM was also clipped. Due to 95 categories of business being represented

311 in the data, attempts had to be made to reduce the sparsity in the data to improve

312 performance. These Places data were aggregated into 14 broad categories: amusement,

313 car, drink, emergency, food, contractor, leisure, medical, office, public building, retail,

314 service, transport, and storage. A summary of all type categories before and after 
315 reduction can be found in Table A.2 Supplemental materials (appendix A). The places

316 type data was then converted into one-hot-vector variables for categorical data to be 317 represented in the model.

\subsubsection{Demographic and Crime Data}

319 Demographic features were used in this study due to their inclusion in previous building

320 fire prediction research (Madaio et al., 2016; Walia et al., 2018). Demographic data was collected and released by the ONS and accessed from EDINA DigiMap as data attributed to output area shapefiles (Office for National Statistics, 2016; Edina, 2019). The census data used was released in 2011 and is the most recent census data available (Office for

324 National Statistics, 2011). Data relating to age, employment, education, social class, 325 residency, tenancy, and ethnicity was collected for the study in order to evaluate their contribution to classification performance. Crime data was included in this study due to its usage in prior research (Madaio et al., 2016; Walia et al., 2018). Crime data was collected by the Metropolitan Police service and accessed from the London data store (London Data Store, 2019). Crime records represent numbers of crime incidents at the

330 lower super output level (Office for National Statistics, 2016). Crime rates for 2016 were used as this interval represents the period in the middle of the incidents used. Population data was also collected and used to derive crime rates from the crime totals.

333 The demographic data came in the form of output area shapefiles of ONS output areas

334 attributed with demographic variables. The attribute tables were filtered for series that 335 were relevant to the application before spatial joins were performed to attribute building 336 shapefiles with the desired variables. Crime total data was acquired in the form of tables 337 of crime rate data organised by lower super output area (LSOA). LSOA crime values 338 were divided by LSOA population totals to produce crime rates. A shapefile of London 339 LSOAs was downloaded, and crime data was joined to produce geographic extents of 
340 crime rate values. This was then spatially joined to the MM buildings to pass on the

341 desired attributes.

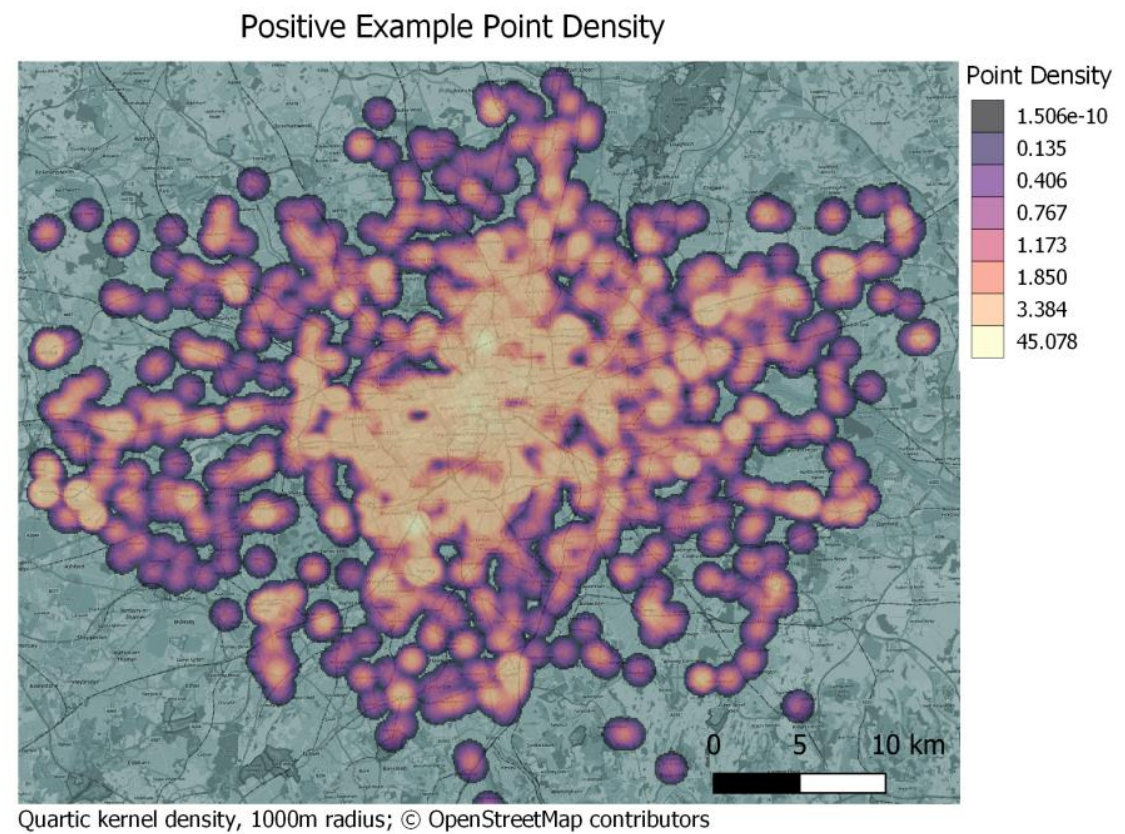

Negative Example Point Density

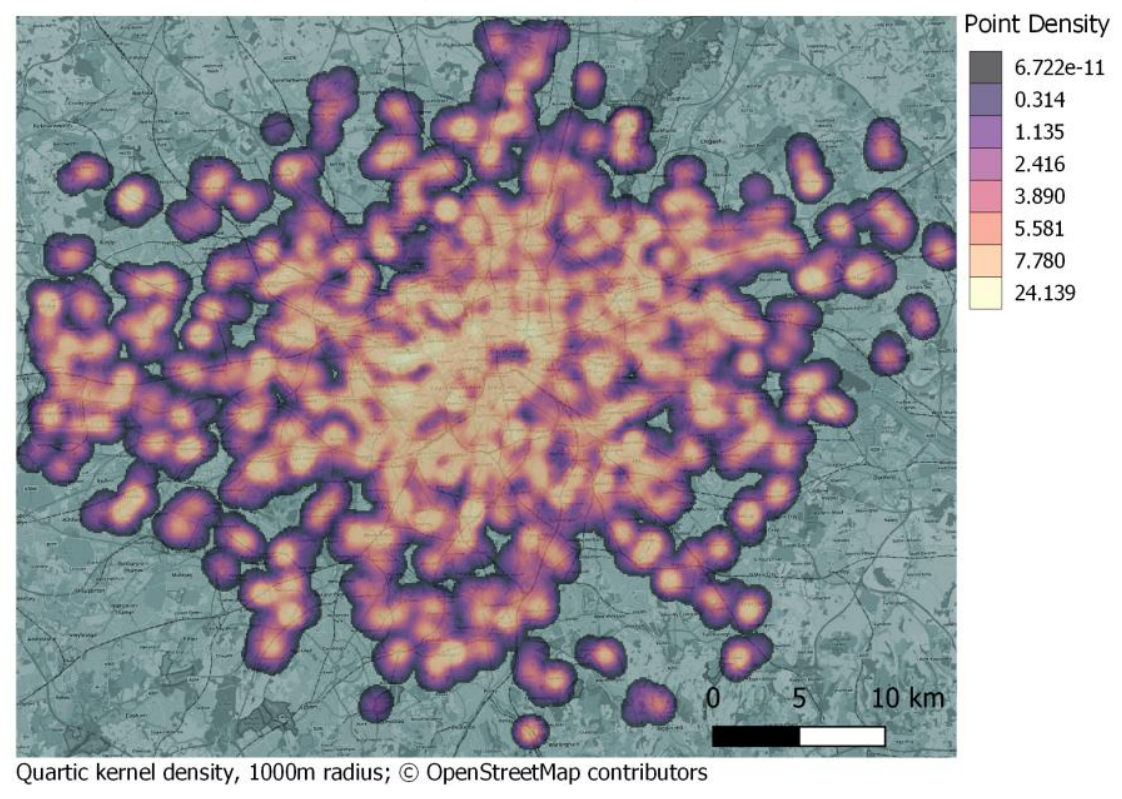

343 Figure 7: Spatial distribution of datasets for positive and negative classes

\section{2.1.6 Final Data}

345 After elimination of data entries that were missing features, a total of 6690 examples

346 remained in the final dataset. Within the final data were 2087 positive examples and 4603

347 negative examples of building fire, giving a ratio of 0.312: 0.688 to be used for class 
348 weighting. The spatial distributions of the classes can be found in Figure 7. All data series

349 apart from the aerial imagery and DSM were put into the same data table and normalised

350 in preparation to be inputted to the model. The image and DSM files were put into their

351 own directory. The image data was also normalised before being used in modelling. A

352 training-validation-test split of 80:10:10 was used.

\section{$353 \quad 2.2$ Modelling}

354 The PyTorch package was chosen as the framework for using ANN in this study due to 355 general usability, ease at which it can be implemented, GPU Capability and its wide range 356 of features (PyTorch, 2019).

\subsubsection{Architecture}

358 An initial architecture compatible with the input data types had to be chosen upon which hyperparameters could be tested. Two CNNs (Figure 8, a,b) would each take an image input then a standard ANN would take the tabular data and run it through a series of fully

361 connected layers before being concatenated to the outputs of the convolutional layers

362 (Figure 8c). This would then go through a final fully connected layer before classification.

363 The path of each data input through the model will be referred to as a branch (e.g. the

364 image branch). This architecture is illustrated in Figure 8 and 9. The benefit of having a

365 hybrid model is that the algorithm can make a classification based on multiple sources of 366 data that are of different types (Audebert et al., 2019). This allows the model to optimise 367 itself across the different data sources. 

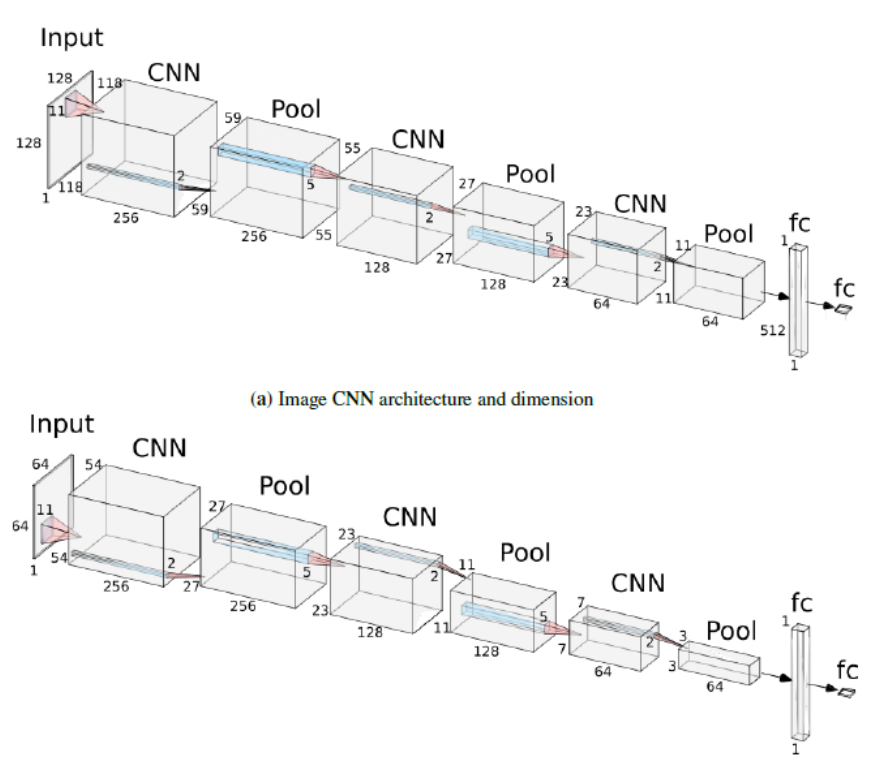

370

371 Figure 8: Diagrams summarising model architecture: The output of CNN image branches (a) and

(b) are concatenated to the output of a fully connected layer of the vector branch before a final

373 fully-connected layer (c), produced using NN-SVG (LeNail, 2019) rather than make separate

374 models that each make an individual classification.

375

376 Figure 9: A schematic diagram of the model architecture chosen after hyperparameter testing.

$377 \mathrm{CNN}=$ Convolutional neural network, MLP=Multi-layer perceptron, fc=Fully connected layer (a)

378 Image CNN architecture and dimension, (b) DSM CNN architecture and dimension, (c)

Concatenation

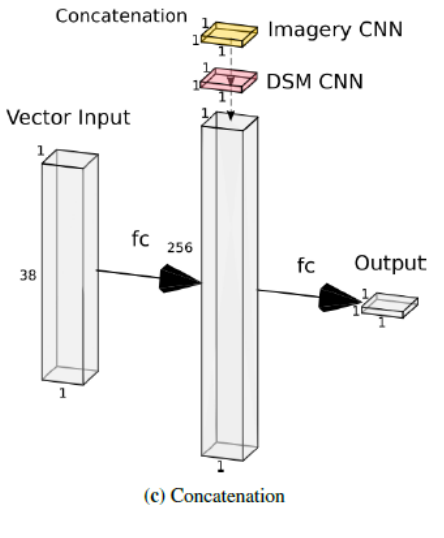


382 Preliminary testing of the $\mathrm{CNN}$ branches as individual models found that by having a

$38311 \times 11$ convolution kernel size in the first CNN layer led to an $13 \%$ reduction in average

384 validation error when conducted over 10 sets of training. This was used as it may be that

385 the larger kernel is able to recognise larger objects within the images. The other

386 convolution kernel sizes were kept at $5 \times 5$. The increased size of the first convolution

387 kernel is also seen in the architecture of AlexNet (Krizhevsky et al., 2012).

\subsubsection{Loss Function}

389 The classes of the dataset being used in this study are unbalanced and so a loss function

390 that takes this into account is used so that each class is treated equally. The binary cross-

391 entropy loss function is used as, along with the model prediction and target, this function

392 also takes in a weighting of each example (Phan et al., 2006). The values 0.688 and 0.312

393 were used for the positive and negative classes, respectively. These are proportionate to

394 the class imbalance so that the model will not just attempt to optimise with respect to the

395 majority class.

\section{$396 \quad$ 2.2.3 Training Evaluation}

397 Model training is achieved by inputting a dataset to the model and mapping this input to

398 a desired target output. The error between the target output and prediction will usually

399 improve with number of epochs taken however it is not a good indicator of model

400 performance as the model has seen the training data before and may eventually learn the

401 subtleties of the training data perfectly, thus overfitting the data. By testing performance

402 against an unseen validation data set, the model's ability to generalise can be investigated.

403 This gives a better indication as to whether there is a correlation in the data used to make

404 predictions and the actual target the model is attempting to predict. Loss was tested 
against the validation data on every $32^{\text {nd }}$ batch during training. This was done over 128

406 data samples, close to the maximum number that could be accommodated on the GPU.

407 All pre-processing stages were conducted on a Dell XPS 9550 laptop with an intel i7 processor. A computer running a Nvidia Titan RTX was connected to via SSH for running parallel processing in the methods used to train the ANNs.

410 Pre-processing was carried out on a Windows 10 operating system with Python 3.7 using

411 PyCharm 2019.2 as the IDE, while Jupyter Lab was used for SSH. Python packages used

412 in the study are summarised in Table A.1 in Supplemental materials, appendix A while

413 scripts used can be found in appendix B.1.

\subsubsection{Hyperparameter Optimisation}

415 Overall, 162 combinations of hyperparameters were tested. The hyperparameters tested 416 are summarised in Table 1. Due to the complexity of the architecture of the model used, 417 simplifications have been made to the range of potential hyperparameters that could be tested. In each branch a starting number of nodes was specified for the first hidden layer

419 then this value was halved with each succeeding hidden layer. Furthermore, the 420 hyperparameters associated with the CNNs were both altered in unison to further reduce 421 the number of required hyperparameter permutations. The rectified linear unit function was used for activation of layers as it offers better performance and generalization when compared to some counterparts (Chigozie et al., 2018). A sigmoid function was used for

424 the output to produce a probability value.

425 Table 1.

426 A learning rate scheduler was implemented whereby an initial rate of 0.005 was halved whenever

427 validation loss did not improve for 7 consecutive epochs. An early dropout was also implemented where if the model did not improve for 20 epochs training would cease. If validation loss was lower than any previous end-epoch value, the model weights were saved, potentially overwriting 
430 a previous epoch's weights. This allowed the highest performing weights to always be preserved.

431 As training was run on a GPU to speed up processing, the batch size was constrained to a 432 maximum of 128 so that there was sufficient memory available to hold all data and weights at 433 any time.

\begin{tabular}{lll} 
Hyperparameter & Description & \multicolumn{1}{l}{$\begin{array}{l}\text { Values } \\
\text { tested }\end{array}$} \\
\hline $\begin{array}{l}\text { Vector start } \\
\text { nodes }\end{array}$ & $\begin{array}{l}64,128, \\
256\end{array}$ \\
\hline & $\begin{array}{l}\text { The number of nodes in the first hidden layer } \\
\text { The number of nodes in the first hidden } \\
\text { CNN start nodes }\end{array}$ & $\begin{array}{l}64,128, \\
\text { convolutional }\end{array}$ \\
\hline \multirow{2}{*}{ Vector layers } & $\begin{array}{l}\text { layers of the image branches } \\
\text { The number of hidden layers the vector data }\end{array}$ & $1,2,3$ \\
\hline \multirow{2}{*}{ CNN layers } & $\begin{array}{l}\text { propagates through before concatenation } \\
\text { The number of convolutional layers the image } \\
\text { data propagates through before concatenation }\end{array}$ & $1,2,3$ \\
\hline Batch size & $\begin{array}{l}\text { The number of training examples to be used } \\
\text { in backpropagation in each epoch }\end{array}$ & 64,128 \\
\hline
\end{tabular}

\subsubsection{Shapley Values}

436 In order to calculate Shapley values, the SHapley Additive exPlanations (SHAP) package

437 (Lundberg and Lee, 2017) is implemented where, for each example put through the 438 model, feature values are altered to observe the impact they have on the model output. It 439 is assumed that by giving a feature a low value the absence of a feature is simulated.

\section{$440 \quad$ 2.2.6 Ablation}

441 Individual features and feature groups were aggregated within the tabular data, 442 summarised in Table A.2 in supplemental materials, and were ablated by excluding the 443 features from training. With the aerial image and DSM CNN branches, each CNN branch 444 architecture had to be excluded along with the feature. For each feature ablated the model 445 was trained 10 times and median validation ROC AUC was used to compare the 
446 performance of the models. The highest performing model from this analysis would then

447 be used as the final model.

448

449

450

451

452

453

454

455

456

457

458

459

460

461

462

463

464

465

466

467

468

469

\subsection{Cost-Benefit Analysis}

Building fire incidents have the potential to inflict high cost of repair and even fatalities when they occur. For this reason, there is motivation in taking preventative measures towards reducing fire risk, such as inspections carried out to ensure that the building complies with fire regulation. The cost of an inspection in conjunction with the potential cost of a fire incident may be combined in order to assess the costs and benefits of mitigation measures, where the resources required to carry out the inspection are thought of as the cost value then the cost of fire that may be avoided will be the benefit value. The cost-benefit ratio can then be used as the acceptable FN/FP ratio in classification, defined below (Sheng and Ling, 2006).

\section{Cost/ benefit $=$ FN/FP (4.2)}

A cost-benefit analysis will be implemented in this study to suggest an optimal classification threshold of the final model in a fire brigade's operational scenario. While the LFB does not publish the cost of a building inspection, a value of $£ 1875$ was averaged over three commercial quotes for the building fire safety inspection of a $230 \mathrm{~m}^{2}$ property, the median size in the dataset used. The average area of fire damage of a non-residential building in the UK was $28.3 \mathrm{~m}^{2}$ in $2018 / 2019$ (Home Office, 2018), while the average cost of fire damage for non-residential buildings is $£ 1405 / \mathrm{m}^{2}$ (Salter et al., 2013). This gives an average fire damage cost of $£ 39,761$. Thus, the desired FN/FP can be calculated.

$$
1875 / 39761=0.047(4.3)
$$

The threshold at which the FN/FP is closest to 0.047 will be used to demonstrate the performance of the final classifier in an operational setting. 


\subsection{Hyperparameter Optimisation}

472 Experimentation of model hyperparameters was conducted to find the best parameters

473 for a model. As training was conducted on an unbalanced dataset the validation loss was

474 used to measure model performance during training. The hyperparameters of the model

475 used are presented in Table 2, while changes in error associated with varying

476 hyperparameters are graphed in (Figure 1 supplemental materials).

477 Table 2: The hyperparameter combination used for the hybrid model in this study

\begin{tabular}{lr} 
Hyperparameter & Value chosen \\
\hline Vector start nodes & 256 \\
\hline CNN start nodes & 128 \\
\hline Vector layers & 1 \\
\hline CNNlayers & 3 \\
\hline Batch size & 128 \\
\hline
\end{tabular}

478

479 The lowest validation loss achieved in hyperparameter testing was 0.225 . The 480 hyperparameter combination of this model is summarised in Table 2 while the losses and 481 accuracy values are shown in Figure 10. The weights of the chosen model were saved 482 after training over 47 epochs, shown by the green vertical line in Figure 10, before training 483 was dropped out after 67 epochs. 

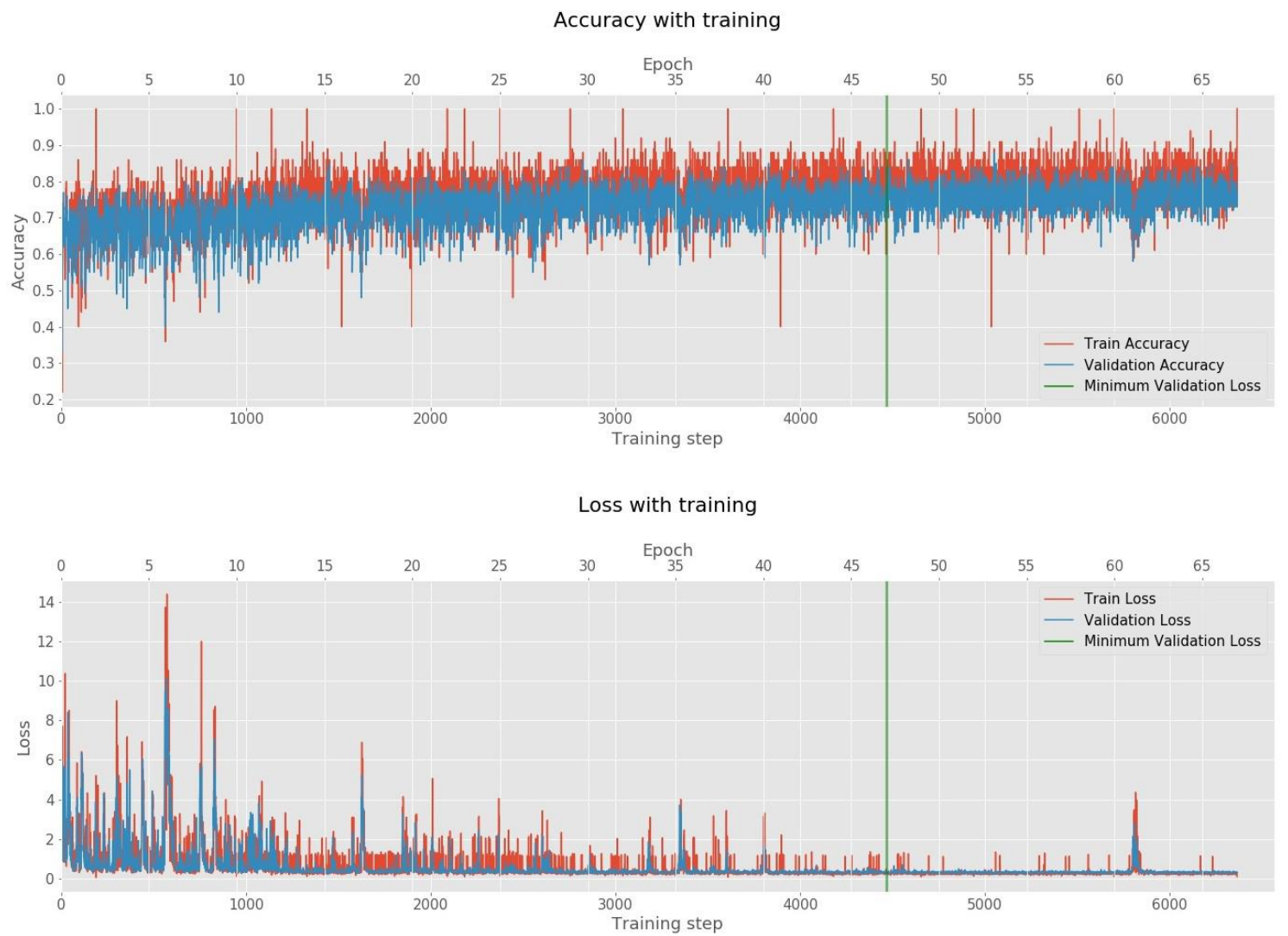

Figure 10. Graph showing changes in loss and accuracy over train and validation sets throughout training

487 It can be seen from Figure 10 that, overall, train accuracy increases and train loss decreases throughout training of the model, suggesting that there is a correlation between the training data and the target that the model is capable of learning. Furthermore, a

490 similar pattern in the validation accuracy and loss suggests that the learned mapping is 491 consistent across the dataset and train performance are not entirely due to overfitting. This 492 model achieved a validation ROC AUC of 0.778.

\section{$493 \quad 3.2$ Shapley values}

494 Shapley values were calculated over the entire training set for all features from the tabular 495 data using the SHAP package. In general, most features show some contribution to the 496 output value. The summary plot in Figure 11 show the SHAP values. Each row on the 497 plot represents a feature of the dataset and each training example is represented by a dot. 
498 The colour of the dot represents the value of the feature while the SHAP value is shown

499 by the dots position on the x-axis. The area feature is shown on its own separately on

500 Figure 12 using a different scale while all other features are shown on Figure 11.

501

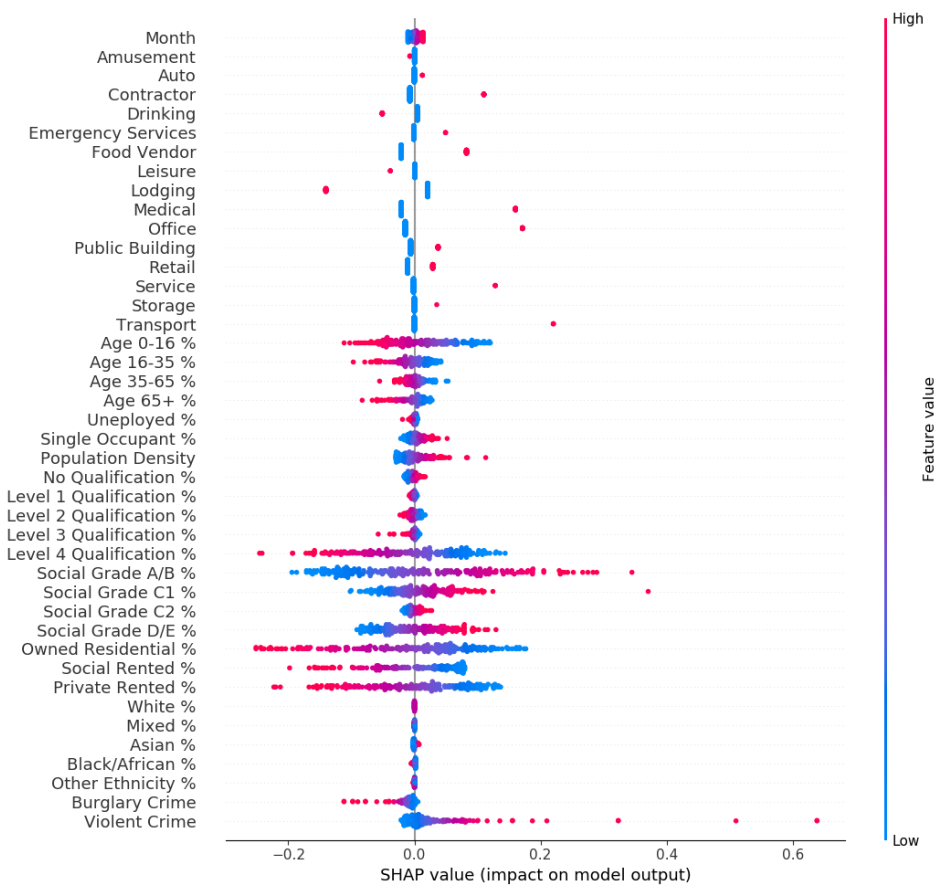

502 Figure 11. SHAP summary plot for all tabular features excluding building area

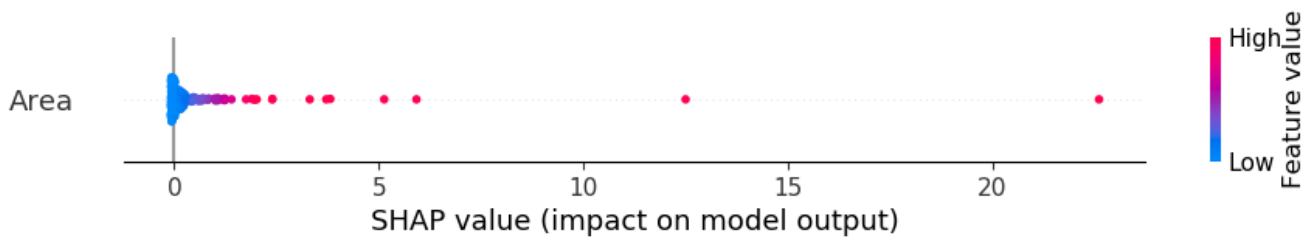

504 Figure 12. SHAP summary plot the building area feature

505 For the area feature, in very few examples, a high feature value heavily influences the

506 target output. Compared to other features, the area feature has a vastly higher mean SHAP

507 value at 5.7, 32 times greater than the second highest mean SHAP value, for violent crime 508 at 0.018 .

\section{$509 \quad 3.3$ Ablation}

510 In the feature ablation portion of this study features were excluded from the dataset,

511 models were trained using the new feature set, and performance was evaluated to gauge 
512 the impact of the individual features on the classification performance of the model. Each

513 model was trained 10 times to get a reliable estimate of performance. Figure 13 shows a

514 boxplot of all ablation AUC results for each group removed. A lower position on the y

515 axis represents a deterioration in classification performance when that feature was

516 removed. The median statistic is chosen for comparison as several groups exhibit a

517 skewed distribution of AUC values.

518 In Figure 13, while models trained excluding some feature groups, such as age, show a 519 narrow range of AUC scores, for other features, such as social class, there is a wide range.

520 Comparisons between median AUC is more easily made by observing differences

521 between the 'all' feature model median AUC (greed dashed line on Figure 13) and the 522 group removal medians (blue lines within boxes). By removing either the age, crime, 523 ethnicity, month, qualification or residential features, the median AUC increases.

524 Conversely, it is seen that the removal of either area, demographics as a whole, the image branch, places or social class lead to a decrease in the median AUC. When the DSM branch is removed there is little change in median AUC.

\section{AUC Boxplot by Feature Group Removed}

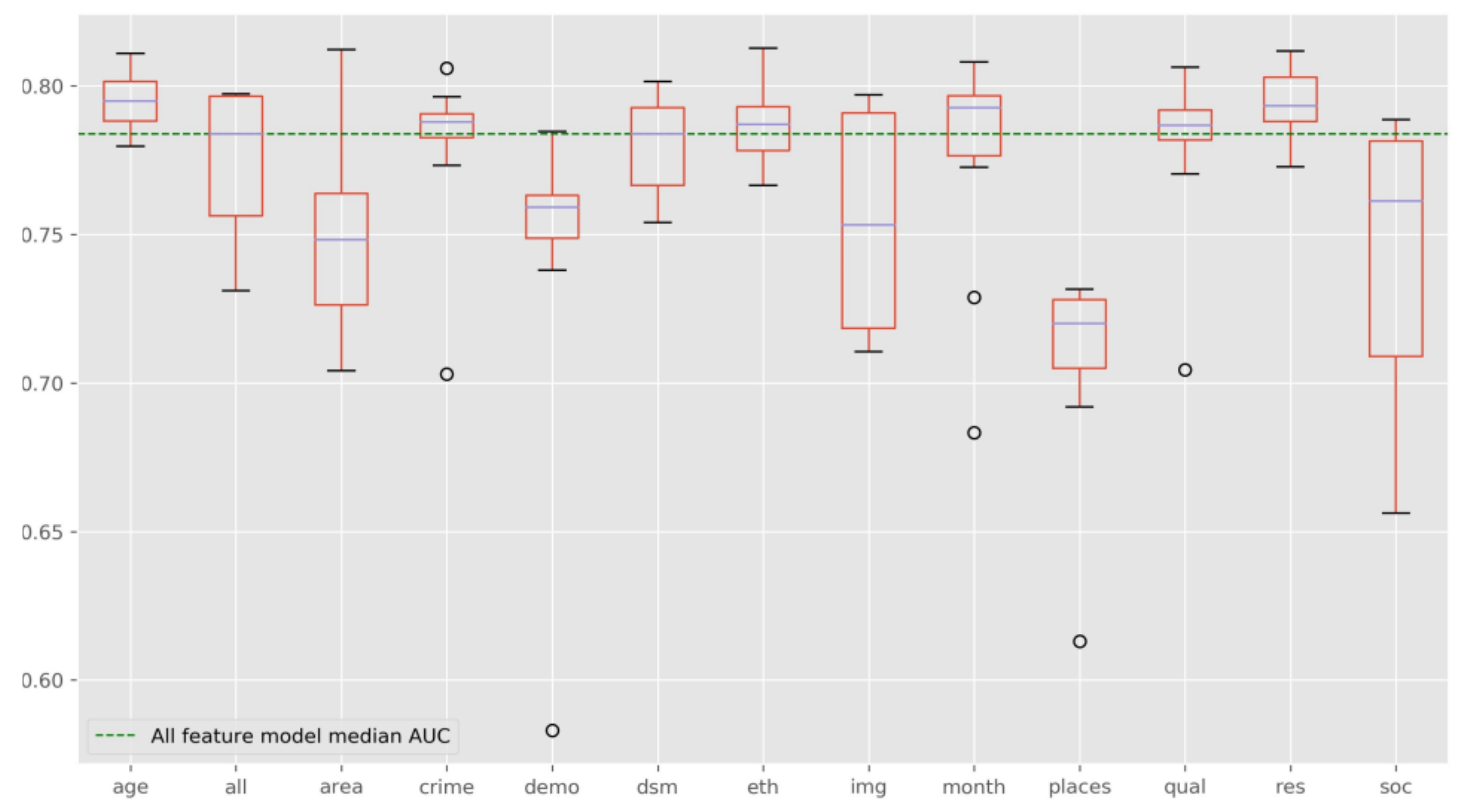

528 Figure 13. Box plot of AUC for models trained on datasets with features removed. The 
$529 \mathrm{x}$-axis shows the feature removed, except for 'all' which is the model trained with the original set 530 of features.

\subsection{Final Model}

532 The highest AUC from the ablation study on the validation set was 0.8128 and was

533 achieved by a model where the ethnicity feature was removed. The final model ROC for

534 validation and test set of this model is presented in Figure 14. The threshold of highest

535 classification performance, observed to be 0.654 , was found by taking the threshold at

536 which the sum of sensitivity and specificity (1-FPR+TPR) was at its maximum. A map

537 of confusion matrix components at this threshold is presented in Figure 15. The threshold

538 at which $\mathrm{FN}=\mathrm{FP}=0: 047$, the cost-benefit ratio, was 0.093 . Tables 3 and 4 show the

539 confusion matrices for highest class separation and cost-benefit thresholds, respectively.

540 The lowest validation loss weights for this mode were saved after 83 epochs at 0.2113

541 validation loss before model training was dropped out after 103 epochs. Figure 15

542 summarises the accuracy and loss over the training and validation data sets throughout

543 training. Diagrams summarising the CNN branches and network architecture are shown

544 in Figure 9. 


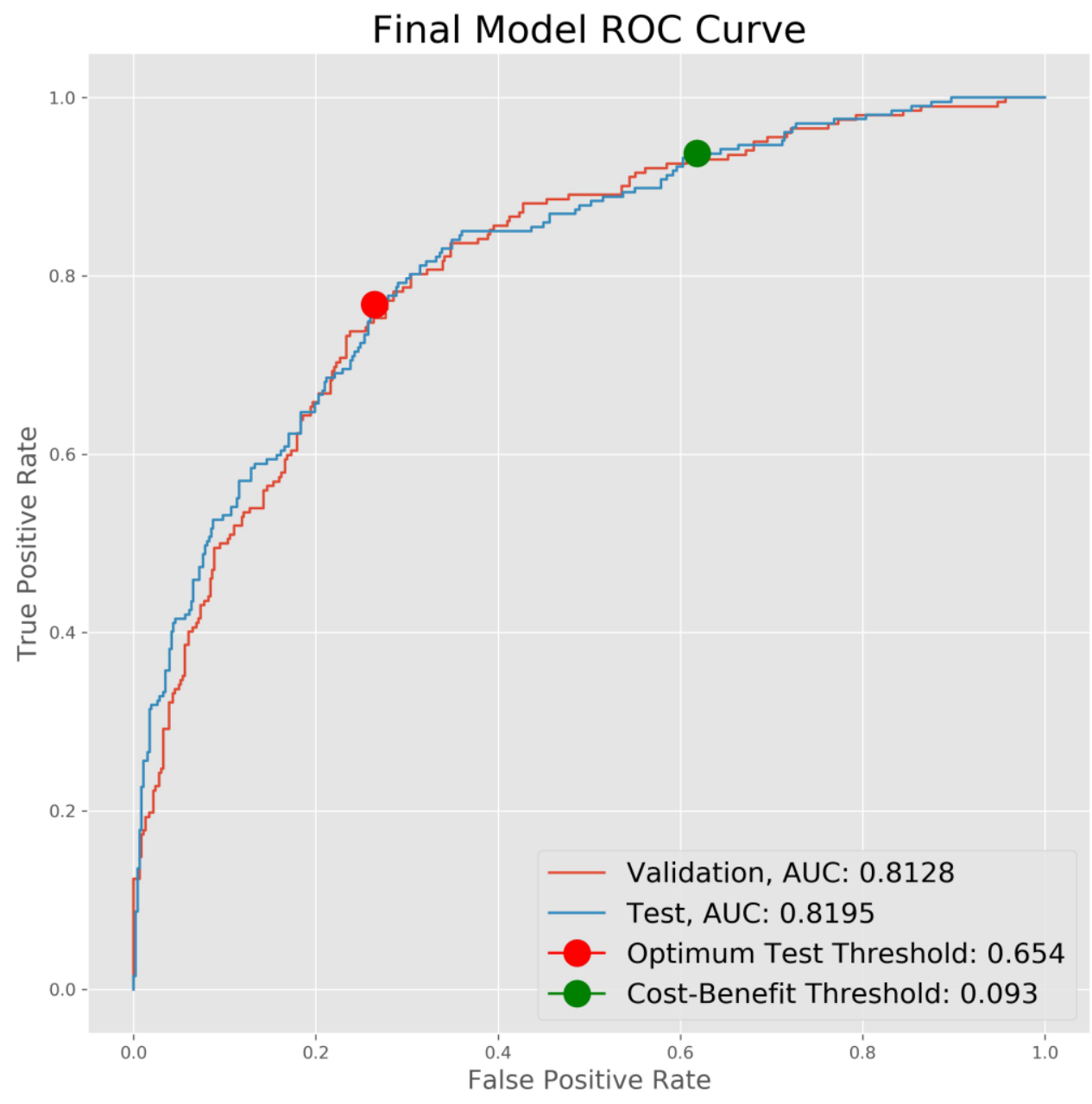

546 Figure 14. ROC curve for final model validation and test classification performance showing

549 Table. 3 Test set confusion matrix for the threshold of max class separation positions of optimum and cost-benefit thresholds

$$
\begin{array}{|rrrr} 
& & \multicolumn{2}{c}{\text { predicted }} \\
& & \text { positive } & \text { negative } \\
& & & \\
\hline \bar{J} & \text { positive } & 129 & 59 \\
\hline \multirow{0}{*}{} & & & \\
& \text { negative } & 106 & 375 \\
\hline
\end{array}
$$

552 Table. 4 Test set confusion matrix for the cost-benefit threshold

$$
\text { predicted }
$$

\begin{tabular}{|rrr|r} 
& & positive & negative \\
\hline$\overline{\widetilde{T}}$ & positive & 194 & 14 \\
\hline \multirow{\pi}{\pi}{} & negative & 285 & 176 \\
\hline
\end{tabular}



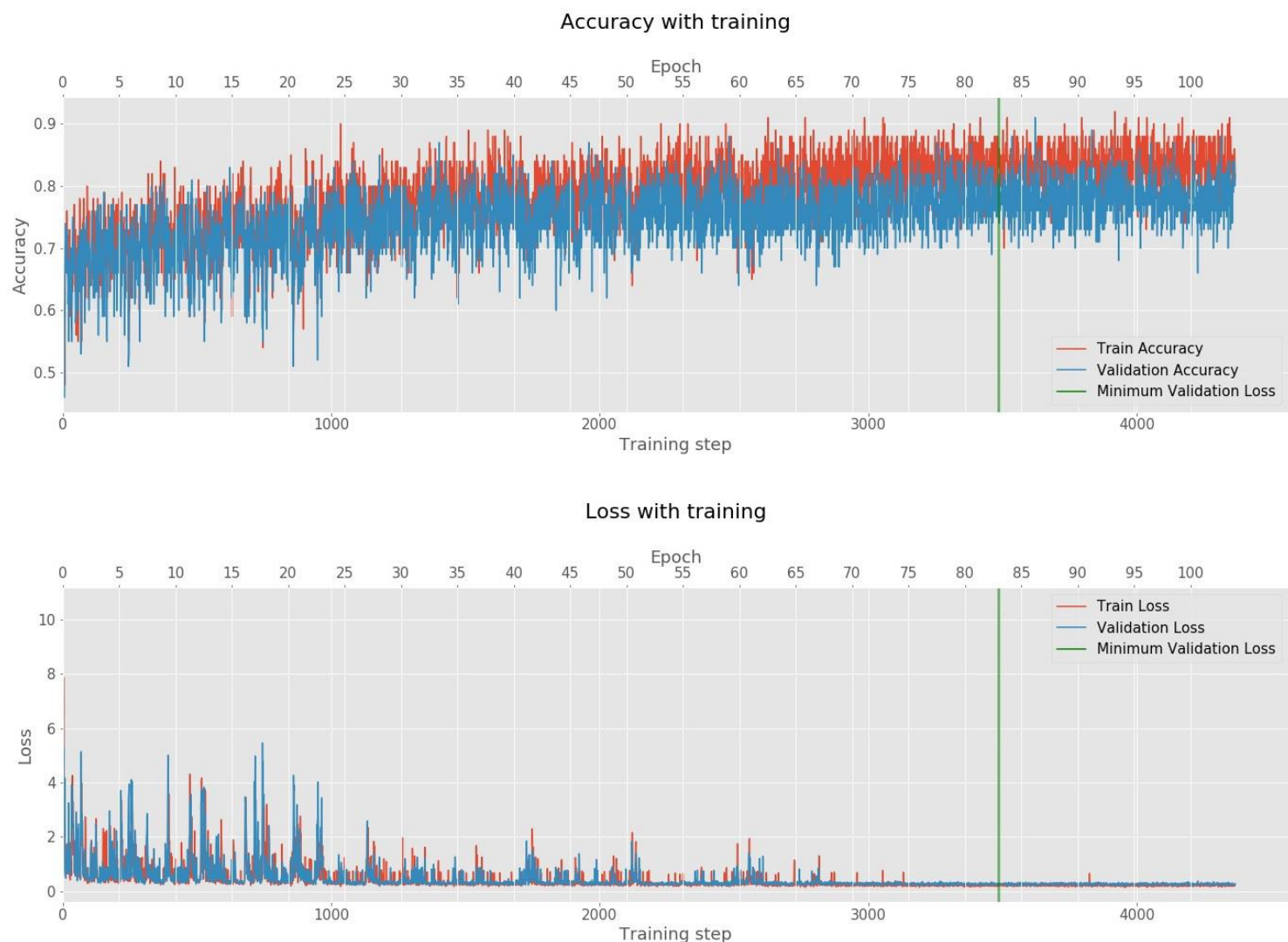

Figure 15. Training summary for the final model

\section{Spatial Distribution of Building Fire Risk Classification}

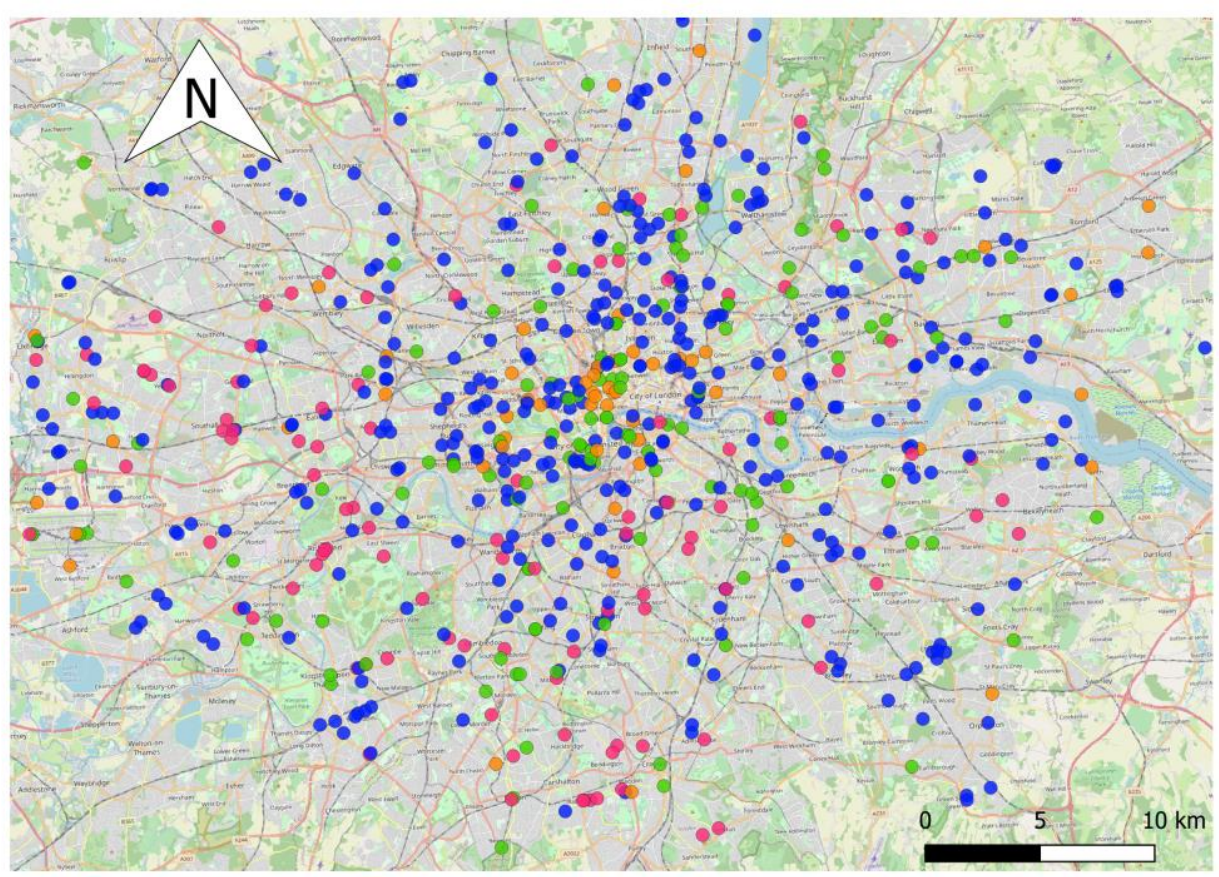

Figure 16. Spatial distribution of test set confusion matrix classification performance terms for a threshold of 0.654 . TP represent the number of true positives in the dataset, TN represent the number of true negative in the dataset, FP represent the number of false positives in the dataset, FN represent the number of false negatives in the dataset

\section{Classification}

TP: 129

- TN: 375

- FP: 106

FN: 59

Threshold: 0.654 


\section{Discussion}

564

565

566

567

568

569

570

571

572

573

574

575

576

577

578

579

580

581

582

583

584

585

586

\subsection{Shapley Values}

The results from the Shapley value investigation represent the impact of feature values on a model output.

\subsubsection{Building Area}

The building area feature had a higher impact on model output than any other feature from the tabular data. Similar results have been found by Madaio et al., (2016) who point out that many of the most important features from their modelling are related to building size for their Random Forest model. While one would expect larger buildings to be generally more susceptible to fire risk as they have potential for more activity to take place within them, it is difficult to conclusively say that this is the reason for the observed correlation. The subject is complicated further by the fact that incident location is not accurate for all building incidents in the LFB data. It may be that there is a greater driving force behind the accurate collection of data relating to larger buildings within which incidents with more casualties or a greater cost of damage may occur. This would lead to larger buildings being over-represented within the positive fire incident class and give a skewed representation of reality.

\subsubsection{Places}

Generally, the places features had a large impact on model outputs. This is a significant finding as it solidifies the idea that the types of activities that occur within a nonresidential building have an impact on the buildings fire risk. This agrees with the regression coefficients described by Madaio, et al. (2016). A higher building fire risk from food establishments may be explained by the cooking activities that are carried out in these establishments as cooking equipment have been shown to be one of the major 
587 sources of building fire ignition (Shai, 2006). This is also supported by Manes and Rush

588 (2018), who found that fire incident rate for food premises in the UK were 1.8 times

589 higher than any other category. It is interesting to note that while the SHAP values for the

590 presence of food vendors were positive, they were negative for the presence drinking

591 (alcohol) establishments. These results suggest that useful fire risk predictors can be made

592 through a distinction between these categories.

593 The presence of medical services within a building also leads to a higher SHAP value.

594 This pattern is seen in the results of Manes and Rush (2018) whose statistical analysis of

595 building fires in the UK from 2014-15 find that fires in hospitals occurred in $2 \%$ of all

596 buildings of that category within the sample, the second highest incidence rate of the

597 categories they observe. High SHAP values have been found to be associated with the

598 presence of offices in buildings in this study. This contrasts with results of Manes and

599 Rush (2018) who find that offices have a $0.3 \%$ incidence rate, second lowest to dwellings.

600 4.1.3 Age

601 For all age group features, generally higher feature values are associated with a decrease

602 in the model output. This seems at face value rather counter-intuitive as these features are

603 proportions, and it was expected that higher fire risk would be associated with higher

604 proportions of some age group. There does appear to be some minor trends, however.

605 For the age group 0-16, representing children, the extremes of this feature were associated

606 with a higher magnitude impact on the model output than the other age features. In

607 particular, the absence of children in the population is associated with the highest model 608 output of age features. 
610 The SHAP values for unemployment show a narrow spread suggesting that there is little

611 impact of employment rates on model output and virtually no contribution to fire risk.

612 This finding is concordant with the results found by Špatenková and Stein (2010), where

613 no link was found between unemployment and fire incidence. A higher proportion of

614 single occupants in the population of an area lead to an increase in SHAP value. A similar

615 finding is also seen in population density.

616 Of the qualification features, level 4 qualification had the potential to have the greatest

617 impact on SHAP values. Higher proportions of level 1, 2, 3 and 4 qualified populations

618 led to a negative model impact, while higher proportions of population with no

619 qualification lead to a slight positive impact on model output. These results may suggest

620 that education levels in the population have an impact on non-residential fire incidence.

621 Higher social class feature values all have a positive impact on model output. The largest

622 impact on model output is seen from the A/B social grade which reaches up to around 0.3

623 and as low as $-0.2 \mathrm{SHAP}$ value. Grades $\mathrm{C} 1$ and $\mathrm{D} / \mathrm{E}$ feature values have a moderate impact

624 on model output, while $\mathrm{C} 2$ has little impact.

625 All ethnic population features have a very low impact on model output when compared

626 to other features analysed. This suggests that they are not a good predictor of non-

627 residential building fire and may cast doubt onto their use in previous studies (Madaio et

628 al., 2016), however the impact is likely to vary for different cities.

629 While high burglary crime values were associated with an increase in model output, the

630 opposite was the case for violent crime where a few high feature value outliers lead to

631 SHAP values around 0.6. This suggests both features may be effective contributors to 632 model performance. 


\section{4.2.1 Places}

635 It was found that the places data was the largest contributor to the classification 636 performance of the dataset. The median AUC decreased by $8 \%$ when the places features 637 were removed, higher than any other feature group removal. This finding also reflects the 638 result of the Places SHAP values. This suggests that, in this model, building use is the 639 most crucial factor to consider when classifying non-residential fire risk and outweighs 640 the importance of social factors. This also highlights a major difference in analysis of 641 building fire risk factors between residential and non-residential properties.

\section{4.2.2 Building Area}

643 The second highest feature removal decrease in median AUC was from the area feature, 644 with a 5\% decrease in median AUC. While the area features removal tended to improve 645 the model, it is worth noting that the second highest performing model from the ablation 646 study had this feature removed at over 0.81 AUC. While it tends to improve the model 647 there is a possibility that removing the area feature can improve classification 648 performance. It may be the case that, while the area feature does provide a useful indicator 649 of potential fire risk, the model has the potential to rely on it heavily as an individual 650 feature. When this is related to the SHAP value results, where the area features impact on 651 model output had the potential to be over 30 times greater than any other feature, it can 652 be seen how the area feature could overshadow the values of other features when making 653 a prediction.

\section{4.2.3 Demographic Features}

655 When all demographic features were removed the median AUC decreased by 3\% 656 suggesting that social factors contribute somewhat to classification performance and are 
657 useful indicators of non-residential fire risk. When the sub-groups are analysed, however,

658 the removal of only the social classes group leads to a decrease in median AUC. Other 659 demographic variables, when treated as their own feature groups, do not provide any 660 increase in classification performance.

\section{4.2.4 Aerial Imagery and DSM}

662 Ablation was also performed on the aerial imagery and DSM CNN branches. It must be noted that while the other ablation results represent the removal of tabular features from

664 the vector branch of the model, the imagery and DSM ablation results are collected by 665 removing the entire $\mathrm{CNN}$ branch of that feature. While the architecture of the model 666 changes slightly with the tabular feature ablation in that the dimension of the inputs 667 change, the architecture is changed more profoundly by removing an entire CNN branch. 668 When the imagery branch was removed there was a decrease in median AUC 6.3. Final 669 Model Performance 47 by $4 \%$, the third highest decrease seen in the ablation study. This 670 suggests that the aerial imagery component of the model is an effective contributor to 671 classification performance. Conversely, the median AUC increased by $0.002 \%$ when the 672 DSM channel was removed, suggesting that it contributed little improvement to 673 classification performance and was not as useful as the imagery branch. There are some 674 fire risk factors of non-residential buildings that can be deciphered visually. These results 675 do not suggest a mechanism for what characteristics of a building image the model uses 676 to come to more accurate classifications. For instance, it may be that like Liu et al. (2017), 677 the model is able to visually assess the construction and maintenance quality.

\section{$678 \quad 4.3$ Final Model Performance}

679 It was found that the removal of the ethnicity feature yielded the highest classification 
680 performance with a validation set AUC of 0.8128 and a test set AUC of 0.8195 . An optimum threshold was found to be at 0.654 where a TPR of 0.768 and FPR of 0.264 was achieved (Figure 14). The final TPR vastly exceeding the FPR suggests that this classifier has good potential to focus inspection efforts to buildings of high risk.

684

685

686

687

688

689

690

691

692

693

694

695

696

697

698

699

700

701

702

703

\subsection{Classification Spatial Distribution}

Figure 16 shows the spatial distribution of confusion matrix terms from a classification at optimum class separation. Generally, the spatial distribution of the test set is like that of the entire dataset (figure 6), with a higher concentration of examples in central London. Although true positives are found in most regions of the study area, there is some clustering of these values around central London.

\subsection{Cost-Benefit Analysis}

This study has demonstrated how the classification threshold may be moved to meet a cost-benefit efficiency level for building inspections. It can be seen in Figure 14 and Table 3 , that the cost-benefit threshold on the final model manages to correctly classify $93.2 \%$ of instances of building fire in the test set, an increase of $36 \%$ when compared to the optimal threshold. This does come at a cost, however, as the threshold would then incorrectly classify $65.7 \%$ of negative examples as being instances of building fire, an increase of $180 \%$ when compared to the optimal threshold. When the sum of sensitivity and specificity (1-FPR+TPR) for the two thresholds are compared it can be seen that the optimal threshold, at 1.466 , is $12 \%$ higher than the cost benefit threshold, at 1.314 . This suggests that, although such an approach enables certain operational requirements to be met, it is by no means the best performing classifier overall.

For instance, municipal fire departments may implement systems whereby, instead of an average area of building, the inspection cost value could be calculated using the building 
area of individual cases. Furthermore, the unit area cost of non-residential buildings varies

705 between occupancy types so an occupancy dependent benefit value may be implemented 706 (Salter et al., 2013).

\section{Conclusion}

This study has presented a non-residential building fire risk prediction methodology based on a hybrid CNN-MLP approach and assessed the effectiveness of some features commonly used in the literature along with novel image features that were previously

711 unexplored. Three key conclusions have been made through this investigation: i) while

712 classification performance may be improved by including an aerial imagery feature of the

713 building to the model via a CNN branch, the inclusion of 1m GSD DSM data to the model

714 showed no improvement, ii) data relating to building use had the greatest impact on 715 classification performance, while demographic data, apart from that regarding social

716 class, did not lend benefit to the model. Such a finding is significant as some existing 717 studies have used a suite of demographic features, iii) spatial analysis of final model 718 classifications suggest that models built over large regions may lead to areas of poor 719 model performance.

720 As future perspectives, whilst the findings of this study answer some questions about use

721 of specific features in building fire risk models, it raises many more about the future of 722 building fire risk classification. As aerial imagery has been found to benefit building fire 723 risk classification, future work should be concerned with investigating other novel 724 features that hold contextual building information.

725 While the $1 \mathrm{~m}$ DSM feature was not beneficial to the classification in this study, there is 726 insufficient evidence to suggest that building geometry is completely irrelevant. Future 727 work should experiment with DSMs at higher resolution to assess any classification 728 potential before it is ruled out entirely. A more rigorous ablation study, potentially 
assessing a wider range of features, in all combinations would yield more conclusive

730 evidence regarding which features are relevant. Furthermore, a comparison of

731 classification models built with the same features for different locations should be

732 explored. Future work should investigate whether temporal evolution of features can aid

733 classification.

734 Acknowledgements: We thank two anonymous reviewers whose comments and 735 suggestions helped improve on earlier drafts of the manuscript.

\section{References}

737 - Alidoost F., and Arefi H. A CNN-based approach for automatic building detection

738 and recognition of roof types using a single aerial image. PFG - Journal of

739 Photogrammetry, Remote Sensing and Geoinformation Science, 86:235-248, 12

$740 \quad 2018$.

- Audebert N., Herold C., Slimani k., and Vidal C. Multimodal deep networks for text and image-based document classification. arXiv:1907.06370 [cs], 072019.

- Bryant S., and Preston I. Focus on trends in fires and fire- related fatalities, 2017.

- Chigozie E., Nwankpa W., Ijomah A., Gachagan S., and Marshall. Activation functions: Comparison of trends in practice and research for deep learning, 2018.

- Clare J., Garis L., Plecas D., and Jennings C. Reduced frequency and severity of residential fires following delivery of fire prevention education by on-duty fire fighters: Cluster randomized controlled study. Journal of Safety Research, 43:123-128, 2012.

- Collins, L., McCarthy, G., Mellor, A., Newell, G., \& Smith, L. (2020). Training data requirements for fire severity mapping using Landsat imagery and random forest. Remote sensing of environment, 245 
- DaCosta, M., Krinsley, J., \& Abelson, B. (2015). Optimizing Local Smoke Alarm Inspections with Federal Data. Bloomberg Data for Good Exchange (2015).

- Edina. Digimap. https://digimap.edina.ac.uk/, 2019.

- Environment Agency. Lidar composite DSM 2017 - 1m. https://data. gov.uk/dataset/80c522cc-e0bf-4466-8409-57a04c456197/ lidar-composite-dsm-2017-1m, 032020.

- Garis L., and Clare J. A dynamic risk-based framework for redesigning the

- Getmapping. Getmapping - uk aerial photography. https://www. getmapping.com/, 2019.

- Geiß, C., Schrade, H., Aravena Pelizari, P. and Taubenböck, H.: Multistrategy ensemble regression for mapping of built-up density and height with Sentinel-2 data, ISPRS J. Photogramm. Remote Sens., 170, 57-71, doi:10.1016/j.isprsjprs.2020.10.004, 2020

- Google. Overview — places api. https://developers.google.com/places/webservice/overview, 2020.

- The Home Office. Detailed analysis of fires attended by fire and rescue services. https://assets.publishing.service.gov.uk/government/uploads/system/uploads/atta chment_data/file/831136/detailed-analysis-fires-attended-fire-rescue-england1819-hosb1919.pdf, 2018.

- The Home Office. Fire statistics definitions incident recording system (irs). https://assets.publishing.service.gov.uk/government/uploads/system/uploads/atta chment_data/file/610453/fire-statistics-definitions.pdf, 2020. 
- Hong S.,and Jeong S.R. Development and comparison of data mining-based prediction models of building fire probability. Journal of Internet Computing and Services, 19:101-112, 122018.

- Kim, D., Liong, S.-Y., Gourbesville, P. and Liu, J.: An Innovative DEM Improvement Technique for Highly Dense Urban Cities, pp. 229-240, Springer, Singapore., 2020a.

- Kim, D. E., Liong, S.-Y., Gourbesville, P., Andres, L. and Liu, J.: Simple-YetEffective SRTM DEM Improvement Scheme for Dense Urban Cities Using ANN and Remote Sensing Data: Application to Flood Modeling, Water, 12(3), 816, doi:10.3390/w12030816, 2020b.

- Krizhevsky A., Sutskever I., and Hinton G.E. Imagenet classification with deep convolutional neural networks. 2012.

- Kuester K., Mittnik S., and Paolella M.S. Value-at-risk prediction: A comparison of alternative strategies. Journal of Financial Econometrics, 4:53-89,01 2006.

- Law S., Brooks Paige, and Chris Russell. Take a look around: Using street view and satellite images to estimate house prices. ACM Transactions on Intelligent Systems and Technology, 18, 2018.

- Law S., Seresinhe C.I, Shen Y., and Gutierrez-Roig M. Street-frontage-net: urban image classification using deep convolutional neural networks. International Journal of Geographical Information Science, 34:681-707, 122018. large-scale evaluation of the qualities of the urban environment. Computers, Environment and Urban Systems, 65:113-125, 092017. 
- London Data Store. Recorded crime: Geographic breakdown - London datastore. https://data.london.gov.uk/dataset/recorded_crime_summary, 2019.

- London Fire Brigade. London fire brigade incident records - London datastore. https://data.london.gov.uk/dataset/london-fire-brigade-incident-records, 2011.

- London Fire Brigade. Firefighters or officers visiting your building. https://www.london-fire.gov.uk/about-us/services-and-facilities/techniques-andprocedures/firefighters-visiting-your-building/, 2020.

- Lundberg S.M., and Lee S.I. A unified approach to interpreting model predictions, 2017.

- Madaio M., Chen S.T., Haimson O., Zhang W., Cheng X., Hinds-Aldrich M., Chau D., and Dilkina B. Firebird: Predicting fire risk and prioritizing fire inspections in Atlanta, 2016.

- Manes M., and Rush D. A critical evaluation of bs pd 7974-7 structural fire response data based on USA fire statistics. Fire Technology, 55:1243-1293, 10 2018.

- Mitri G., Jazi M., and McWethy D. Assessment of wildfire risk in Lebanon using geographic object-based image analysis, 062015.

- Monfort D., Negulescu C., Belvaux M., Remote sensing vs. field survey data in a post-earthquake context: Potentialities and limits of damaged building assessment datasets, Remote Sensing Applications: Society and Environment, Volume 14, 2019, Pages 46-59, ISSN 2352-9385,

- Office for National Statistics. 2011 census - office for national statistics. https: //www.ons.gov.uk/census/2011census, 2011.

- Office for National Statistics. Census geography - office for national statistics. https://www.ons.gov.uk/methodology/geography/ 
ukgeographies/censusgeography, 2016.

- Oliveira S, Zêzere JL. Assessing the biophysical and social drivers of burned area distribution at the local scale. J Environ Manage. 2020 Jun 15; 264:110449

- Ordnance Survey. Os mastermap topography layer - Great Britain's landscape vector map data. https://www.ordnancesurvey.co.uk/businessgovernment/products/mastermap-topography, 2020.

- Phan H., Yamamoto K., Phan T., and Yamamoto K. Resolving class imbalance in object detection with weighted cross entropy losses, 2006.

834 - Preuschoff K., Quartz S.R., and Bossaerts P. Human insula activation reflects risk prediction errors as well as risk. Journal of Neuroscience, 28:2745-2752, 032008.

B. Times investigation: Thousands of large L.A. buildings are long overdue for fire inspections. Los Angeles Times, 06 2015. PyTorch. Pytorch. https://pytorch.org/, 2019.

839 - Salter C., Ramachandran G., Emmitt S., and Bouchlaghem N. Economic cost of 840 fire: Exploring UK fire incident data to develop a design tool. Fire Safety Journal, 62:256-263, 112013.

- Shai D. Income, housing, and fire injuries: A census tract analysis. Public Health Reports, 121:149-154, 032006.

- Shapley L.S. Stochastic games. Proceedings of the National Academy of Sciences, 39:1095-1100, 101953.

- Sheng V., and Ling C. Thresholding for making classifiers cost sensitive, 2006. distribution of domestic fires. International Journal of Geographical Information Science, 24:841-858, 042010. 
- Schunder T., Yin D., Bagchi-Sen S., Rajan K., A spatial analysis of the development potential of rooftop and community solar energy, Remote Sensing Applications: Society and Environment, Volume 19, 2020, 100355, ISSN 23529385 ,

- Wang H., Zhao T., Li L.C., Pan H., Liu W., Gao H., Han F., Wang Y., Qi Y, and Liang Z. A hybrid CNN feature model for pulmonary nodule malignancy risk differentiation. Journal of X-Ray Science and Technology, 26:171-187, 012018. the united states final report by, 2017.

860 